Universidad Católica del Norte

Antofagasta - Chile

\title{
Existence and multiplicity of positive unbounded solutions for singular BVPs with the $\phi$-Laplacian operator on the half line
}

\author{
Chahira Attia \\ University of Boumerdes, Algeria \\ Salima Mechrouk \\ University of Boumerdes, Algeria \\ and \\ Ouiza Saifi \\ Algiers University 3, Algeria \\ Received: January 2021. Accepted : July 2021
}

\begin{abstract}
We provide in this work sufficient conditions for existence and multiplicity of positive unbounded solutions for a class of singular second-order boundary value problem associated with a $\phi$-Laplacian operator and posed on the half-line. The proofs are based on a theorem of cone expansion and compression in a Banach space.
\end{abstract}

Key words: Cones, second order, unbounded solution, singular problem, fixed point theory, positive solution, BVPs.

2010 Mathematics Subject Classifications: 47H10, 47H11, $34 B 15$. 


\section{Introduction}

Many applied problems modeling various phenomena in physics, epidemiology, combustion theory and mechanics (see, e.g., [2] and the references therein) are governed by boundary value problems (bvps for short) posed on the half-axis $[0,+\infty)$; we quote for instance the propagation of a flame in a long tube.

Because of their mathematical and physical interest, the study of second order differential equations posed on the half line and subject to various boundary conditions have received a great deal of attention during the latter two decades; see [6]-[11] and references therein.

The positivity of solutions is interpreted by the fact that physical parameters and unknowns such as temperature, density or displacement, among others, are positive.

The existence of solutions of infinite boundary value problem of second order ordinary differential equations have been studied by many authors; see [6]-[11] and the references therein.

In [11], the author presented some existence of positive bounded and unbounded solutions to the singular boundary value problem

$$
\left\{\begin{array}{l}
-u^{\prime \prime}=f(t, u), \quad t \in(0,+\infty), \\
u(0)=0, t \rightarrow+\infty \lim u^{\prime}(t)=y_{\infty} \geq 0,
\end{array}\right.
$$

where $f \in C\left((0,+\infty) \times(0,+\infty), \mathbf{R}^{+}\right)$may be singular at $t=0$ and $u=0$.

In [9], D. O'Regan et al. consider the Problem (1.2) with $\phi(u)=u$ and $f$ may be singular at $u=0$. They obtain existence and multiplicity results for positive solutions in the functional space constituted by the functions $u \in C\left(\mathbf{R}^{+}, \mathbf{R}^{+}\right)$satisfying $\lim _{t \rightarrow \infty} u(t) /(1+t)=0$ endowed with the norm $\|u\|=\sup _{t \in \mathbf{R}^{+}} \frac{|u(t)|}{1+t}$. Clearly, the choice of this space is motivated by the boundary condition in Problem (1.2), $u^{\prime}(+\infty)=0$ and fortunately, this space provide a good framework where the fixed point index theory or theorems of cone expansion and compression in a Banach space can be used.

The unique disadvantage of this framework is that we know nothing about the boundeness of the obtained positive solutions.

From a mathematical point of view, a differential operator (fractional or integer-order) may be extended to $p$-Laplacian order operators. Notice that the latter is only linear for $p=2$. The case of the $\phi$-Laplacian is again a 
more general extension of the $p$-Laplacian. This is our main motivation to consider in this work such general homeomorphism operator. In this paper, we will consider the more general problem

$$
\left\{\begin{array}{l}
-\left(\phi\left(u^{\prime}\right)\right)^{\prime}(t)=a(t) f(t, u(t)), \quad t \in(0,+\infty), \\
u(0)=0, t \rightarrow+\infty \lim u^{\prime}(t)=0
\end{array}\right.
$$

where $\phi: \mathbf{R} \rightarrow \mathbf{R}$ is an increasing homeomorphism with $\phi(0)=0, \phi \in$ $C^{1}(\mathbf{R}), a:(0,+\infty) \rightarrow \mathbf{R}^{+}$is a measurable function and $f \in C((0,+\infty) \times$ $\left.(0,+\infty), \mathbf{R}^{+}\right)$may be singular at $u=0$ and satisfies

- $\left(H_{1}\right)$ There exists $\lambda, \mu \in \mathbf{R}$ such that $\lambda<0<\mu$ and $N, M>0$ with $0<N \leq 1 \leq M$ satisfying for all $t \geq 0$ and $u \geq 0$

$$
\begin{aligned}
& c^{\mu} f(t, u) \leq f(t, c u) \leq c^{\lambda} f(t, u), \quad 0<c \leq N \\
& c^{\lambda} f(t, u) \leq f(t, c u) \leq c^{\mu} f(t, u), \quad c \geq M .
\end{aligned}
$$

$\begin{aligned} I_{1} & =\int_{0}^{1} z^{\lambda} a(z) f(z, 1) d z<+\infty, \quad I_{2}=\int_{1}^{+\infty} z^{\mu-\lambda}(1+z)^{\mu} a(z) \\ & \lim _{t \rightarrow \infty} \frac{1}{1+t} \int_{1}^{t} \psi\left(\int_{s}^{+\infty} z^{\mu-\lambda}(1+z)^{\mu} a(z) f(z, 1) d z\right) d s=0 .\end{aligned}$

- $\left(H_{3}\right) \lim _{x \rightarrow \infty} x \psi\left(\int_{x}^{+\infty} a(z) f(z, 1) d z\right)=+\infty$.

where $\psi$ denotes the inverse function of $\phi$.

A typical example of weight $a$ and nonlinearity $f$ satisfying $\left(H_{1}\right), I_{1}$ and $I_{2}$ is

$$
a(t)=\frac{1}{(1+t)^{m}} \text { and } f(t, u)=\frac{1}{\sqrt[\nu]{u}(1+t)^{n}}, m, n>\nu>2,
$$

where $\lambda=\frac{-1}{\nu}$ and $\mu=\frac{1}{\nu}$.

Recent papers have also investigated the case of the so-called $p$-Laplacian operator. Existence of three positive solutions for singular $p$-Laplacian problems is obtained by means of the three functional fixed point theorem in [7] and [8].

Note that in general the $\phi$-Laplacian operator is not linear and non homogenous, this makes the study of differential equations involving $\phi$-Laplacian more complicated than the case where $p$-Laplacian. Throughout this paper we assume that:

There exists $\alpha, \beta \in \mathbf{R}$ with $0<\alpha<\beta$ such that $\phi^{-}(t) \phi(x) \leq \phi(t x) \leq \phi^{+}(t) \phi(x)$ for all $x \geq 0$ and $t \geq 0$. 
where $\phi^{+}, \phi^{-}$be the function defined on $\mathbf{R}^{+}$by

$$
\phi^{+}(t)=\left\{\begin{array}{l}
t^{\alpha} \text { if } t \leq 1 \\
t^{\beta} \text { if } t \geq 1
\end{array} \quad \phi^{-}(t)=\left\{\begin{array}{l}
t^{\beta} \text { if } t \leq 1 \\
t^{\alpha} \text { if } t \geq 1
\end{array}\right.\right.
$$

Typical examples of a functions f satisfying (1.3) are $\phi(u)=u^{p_{1}}+u^{p_{2}}$ where $p_{1}, p_{2}>0$.

It follows from (1.3) that for all $t \geq 0$ and $x \geq 0$

$$
\psi^{-}(t) \psi(x) \leq \psi(t x) \leq \psi^{+}(t) \psi(x)
$$

Where $\psi^{+}, \psi^{-}$be the function defined on $\mathbf{R}^{+}$by

$$
\psi^{+}(t)=\left\{\begin{array}{l}
t^{\frac{1}{\beta}} \text { if } t \leq 1 \\
t^{\frac{1}{\alpha}} \text { if } t \geq 1
\end{array} \quad \psi^{-}(t)=\left\{\begin{array}{c}
t^{\frac{1}{\alpha}} \text { if } t \leq 1 \\
t^{\frac{1}{\beta}} \text { if } t \geq 1
\end{array}\right.\right.
$$

We mean, by a non-negative solution to problem (1.2), a function $u \in$ $C^{1}\left(\mathbf{R}^{+}, \mathbf{R}\right)$ with $\phi\left(u^{\prime}\right) \in C^{1}((0,+\infty), \mathbf{R})$ and $u\left(t_{0}\right)>0$ for some $t_{0}>0$ satisfying all equation in (1.2). We will examine and provide sufficient conditions to obtain a unbounded positives solutions and in turn existence of at least one and twin solution. In contrast, the method taken is via theorem of cone expansion and compression in a Banach space unlike that of [4] who uses fixed point index theory on cones of Banach spaces and the upper and lower solution technique to obtain just existence of solutions for boundary value problem (1.2) where the singularity of the nonlinearity is treated by regularization and approximation. The paper is organized as follows. In section 2, we recall first, some lemmas where we present a fixed point formulation of BVP (1.2). In section 3, we present our main results and their proofs and it is ended by illustrative examples to the theoretical results.

\section{Preliminaries}

In this section, we gather together some definitions and lemmas we need in the sequel.

\subsection{Auxiliary results}

Definition 2.1. A nonempty subset $P$ of a Banach space $E$ is called a cone if it is convex, closed and satisfies the conditions

i) $\quad \alpha x \in P$ for all $x \in P$ and $\alpha \geq 0$

ii) $x,-x \in P$ implies that $x=0$. 
Definition 2.2. A mapping $T: E \rightarrow E$ is said to be completely continous if it is continous and maps bounded sets into relatively compact sets.

Theorem 2.3. ([5], p94) Let $E$ be a Banach space and $P$ be a cone of $E$. Assume $\Omega_{1}, \Omega_{2}$ are open bounded subsets of $E$ with $0 \in \Omega_{1} \subset \bar{\Omega}_{1} \subset \Omega_{2}$ and let $T: P \cap\left(\bar{\Omega}_{2} \backslash \Omega_{1}\right) \rightarrow P$ be a completely continous operator such that either

i) $\quad\|T u\| \leq\|u\|$ for all $u \in P \cap \partial \Omega_{1}$ and $\|T u\| \geq\|u\|$ for all $u \in P \cap \partial \Omega_{2}$,

ii) $\quad\|T u\| \leq\|u\|$ for all $u \in P \cap \partial \Omega_{2}$ and $\|T u\| \geq\|u\|$, for all $u \in P \cap \partial \Omega_{1}$.

Then $T$ has at least one fixed point in $P \cap\left(\bar{\Omega}_{2} \backslash \Omega_{1}\right)$.

In all this paper $E$ denotes the Banach space defined

$$
E=\left\{u \in C\left(\mathbf{R}^{+}, \mathbf{R}\right): \lim _{t \rightarrow \infty} \frac{u(t)}{1+t}=0\right\}
$$

equipped with the norm $\cdot_{E}$, defined for $u \in E$ by $u_{E}=t \geq 0 \sup \left|\frac{u(t)}{1+t}\right|$.

In order to prove the compactness of some operator we will use the following Lemma. Let

$$
C_{l}\left(\mathbf{R}^{+}, \mathbf{R}\right)=\left\{x \in C\left(\mathbf{R}^{+}, \mathbf{R}\right): \lim _{t \rightarrow \infty} x(t) \text { exists }\right\}
$$

Endowed with the norm $x_{l}=t \geq 0 \sup |u(t)|$ this is a Banach space. We recall a classical compactness criterion:

Lemma 2.4. ([3], $p$ 62) Let $M \in C_{l}\left(\mathbf{R}^{+}, \mathbf{R}\right)$. Then $M$ is relatively compact in $C_{l}\left(\mathbf{R}^{+}, \mathbf{R}\right)$ if the following conditions hold:

- $M$ is uniformly bounded in $C_{l}\left(\mathbf{R}^{+}, \mathbf{R}\right)$.

- The functions belonging to $M$ are almost equicontinuous on $\mathbf{R}^{+}$, i.e. equicontinuous on every compact interval of $\mathbf{R}^{+}$.

- The functions from $M$ are equiconvergent, that is, given $\varepsilon>0$, there corresponds $A(\varepsilon)>0$ such that $|x(t)-x(+\infty)|<\varepsilon$ for any $t \geq A(\varepsilon)$ and $x \in M$.

We easily deduce 
Lemma 2.5. Let $M \subseteq E$. Then $M$ is relatively compact in $E$ if the following conditions hold

a) $M$ is bounded in $E$.

b) The functions belonging to $\left\{u: u(t)=\frac{x(t)}{1+t}, x \in M\right\}$ are locally equicontinous on $\mathbf{R}^{+}$, that is equicontinous on every compact interval of $\mathbf{R}^{+}$.

c) The functions belonging to $\left\{u: u(t)=\frac{x(t)}{1+t}, x \in M\right\}$ are equiconvergent at $+\infty$, that is given $\varepsilon>0$, there corresponds $A(\varepsilon)>0$ such that: $|x(t)-x(+\infty)|<\varepsilon$ for any $t \geq A(\varepsilon)$.

\section{Main results}

Throughout this paper, $P$ is the cone of $E$ given by

$$
P=\{u \in E: u \geq 0 \text { and } u \text { is concave in }(0,+\infty)\} .
$$

Consider the operator $T: P \rightarrow P$ defined for $u \in P$ by

$$
T u(x)=\int_{0}^{x} \psi\left(\int_{s}^{+\infty} a(z) f(z, u(z)) d z\right) d s .
$$

Lemma 3.1. If $u \in P$, then $u$ is nondecreasing on $\mathbf{R}^{+}$.

\section{Proof.}

Let $t_{1}, t_{2} \in \mathbf{R}^{+}$be such that $t_{1}<t_{2}$ and $\xi=t_{2}-t_{1}$. Since $u$ is nonnegative and concave, then for all $n \in \mathbf{N}^{\star}$, we have

$$
\begin{aligned}
u\left(t_{2}\right)=u\left(t_{1}+\xi\right) & =u\left(\left(1-\frac{1}{n}\right) t_{1}+\frac{1}{n}\left(t_{1}+n \xi\right)\right) \\
& \left.\geq\left(1-\frac{1}{n}\right) u\left(t_{1}\right)+\frac{1}{n} u\left(t_{1}+n \xi\right)\right) \\
& \geq\left(1-\frac{1}{n}\right) u\left(t_{1}\right)
\end{aligned}
$$

Therefore

$$
u\left(t_{2}\right) \geq \lim _{n \rightarrow \infty}\left(1-\frac{1}{n}\right) u\left(t_{1}\right)=u\left(t_{1}\right)
$$

and our claim follows.

Lemma 3.2. Let $u \in P$ and $\sigma \in(1,+\infty)$. Then we have

$$
u(t) \geq \frac{1}{\sigma} u_{E}, \forall t \in\left[\frac{1}{\sigma}, \sigma\right] .
$$


Proof. First, since the function $v(t)=\frac{u(t)}{1+t}$ is continuous and satisfies $v(0)=v(+\infty)=0$, then it archieves its maximum at $t_{0}>0$. Then, since $u$ is concave and nondecreasing on $(0,+\infty)$, we have for all $t \in\left[\frac{1}{\sigma}, \sigma\right]$

$$
\begin{aligned}
u(t) \geq t \in\left[\frac{1}{\sigma}, \sigma\right] \min u(t) & =u\left(\frac{1}{\sigma}\right) \\
& =u\left(\frac{\sigma-1+\sigma t_{0}}{\sigma+\sigma t_{0}} \frac{1}{\sigma-1+\sigma t_{0}}+\frac{t_{0}}{\sigma+\sigma t_{0}}\right) \\
& \geq\left(\frac{\sigma-1+\sigma t_{0}}{\sigma+\sigma t_{0}}\right) u\left(\frac{1}{\sigma-1+\sigma t_{0}}\right)+\frac{1}{\sigma+\sigma t_{0}} u\left(t_{0}\right) \\
& \geq \frac{1}{\sigma+\sigma t_{0}} u\left(t_{0}\right) \\
& =\frac{1}{\sigma} u_{E} .
\end{aligned}
$$

Similary of the proof of Lemma on [4], we can proof the following Lemma:

Lemma 3.3. Let $u \in P$, then

$$
u(t) \geq \gamma(t) u_{E}, \quad \forall t \in[0,+\infty)
$$

where

$$
\gamma(t)= \begin{cases}t & t \in[0,1] \\ \frac{1}{t} & t \in(1,+\infty)\end{cases}
$$

Proof. Let $t \in[0,+\infty)$ and distinguish between four cases:

- If $t=0$, then $u(0)=0=\gamma(0) u_{E}$.

- If $t \in(0,1)$, then $\frac{1}{t} \in(1,+\infty)$

$$
u(z) \geq t u_{E}, \forall z \in\left[t, \frac{1}{t}\right] .
$$

For $z=t$, we have $u(t) \geq t u_{E}$.

- If $t \in(1,+\infty)$, then $\frac{1}{t} \in(0,1)$, we have

$$
u(z) \geq \frac{1}{t} u_{E}, \quad \forall z \in\left[\frac{1}{t}, t\right] .
$$

For $z=t, \quad u(t) \geq \frac{1}{t} u_{E}$. 
- If $t=1$. Let $\left(\delta_{n}\right)_{n}$ be a sequence such that $\delta_{n}>1$ and $\delta_{n} n \longrightarrow+\infty \longrightarrow 1$. From the previous case, we have

$$
u\left(\delta_{n}\right) \geq \frac{1}{\delta_{n}} u_{E}, \forall n \geq 1 .
$$

It follows

$$
\lim _{n \longrightarrow+\infty} u\left(\delta_{n}\right) \geq \lim _{n \longrightarrow+\infty} \frac{1}{\delta_{n}} u_{E}=u_{E} .
$$

As a result $u(1) \geq \gamma(1) u_{E}$.

Lemma 3.4. Assume that $\left(H_{1}\right),\left(H_{2}\right)$ and (1.4) hold. Then the operator $T$ is well defined.

Proof. Let $u \in P$ and $v=T u$. For $x \in(0,+\infty)$, we have

$$
v(x)=\int_{0}^{x} \psi\left(\int_{s}^{+\infty} a(z) f(z, u(z)) d z\right) d s>0 .
$$

We show for any fixed $x \in(0,+\infty)$, that the integral $\int_{0}^{x} \psi\left(\int_{s}^{+\infty} a(z) f(z, u(z)) d z\right) d s$ is convergent. We have for all $s \in \mathbf{R}^{+}$

$$
\begin{aligned}
\int_{s}^{+\infty} a(z) f(z, u(z)) d z & \leq \int_{0}^{+\infty} a(z) f(z, u(z)) d z \\
& =\int_{0}^{1} a(z) f(z, u(z)) d z+\int_{1}^{+\infty} a(z) f(z, u(z)) d z .
\end{aligned}
$$

Choose $c>0$ such that $2 c u_{E} \leq N$ and $\frac{1}{c}>M$. Thus $c u(z) \leq N$ for $z \in[0,1]$.

We have by Lemma 3.3

$$
\begin{aligned}
\int_{0}^{1} a(z) f(z, u(z)) d z & =\int_{0}^{1} a(z) f\left(z, \frac{1}{c} c u(z)\right) d z \\
& \leq \int_{0}^{1} c^{\lambda}|u(z)|^{\lambda} a(z) f\left(z, \frac{1}{c}\right) d z \\
& \leq c^{\lambda-\mu} \int_{0}^{1}|u(z)|^{\lambda} a(z) f(z, 1) d z \\
& \leq c^{\lambda-\mu} I_{1} u_{E}^{\lambda} \\
& <\infty .
\end{aligned}
$$

Choose $\delta>0$ sufficiently large such that $\delta u_{E} \geq M$ and $\frac{1}{\delta} \leq N$. Thus $\delta z u(z) \geq M$ and $\frac{1}{\delta z} \leq N$ for $z \geq 1$. Analogously, it follows that

$$
\begin{aligned}
\int_{1}^{+\infty} a(z) f(z, u(z)) d z & =\int_{1}^{+\infty} a(z) f\left(z, \frac{1}{\delta z} \delta z u(z)\right) d z \\
& \leq \int_{1}^{+\infty}(\delta z)^{\mu-\lambda}|u(z)|^{\mu} a(z) f(z, 1) d z \\
& \leq \int_{1}^{+\infty}(\delta z)^{\mu-\lambda}(1+z)^{\mu} u_{E}^{\mu} a(z) f(z, 1) d z \\
& \leq \delta^{\mu-\lambda} I_{2} u_{E}^{\mu} \\
& <+\infty
\end{aligned}
$$


This guaranties that $\int_{s}^{+\infty} a(z) f(z, u(z)) d z$ is convergent for all $s \geq 0$ and since

$$
\int_{0}^{x} \psi\left(\int_{s}^{+\infty} a(z) f(z, u(z)) d z\right) d s \leq x \psi\left(c^{\lambda-\mu} u_{E}^{\lambda} I_{1}+\delta^{\mu-\lambda} u_{E}^{\mu} I_{2}\right)<\infty .
$$

Then the integral $\int_{0}^{x} \psi\left(\int_{s}^{+\infty} a(z) f(z, u(z)) d z\right) d s$ is convergent for all $x \in(0,+\infty)$.

Furthermore, we have for all $t \in(1,+\infty)$

$$
\begin{aligned}
\frac{v(t)}{1+t} & =\frac{1}{1+t} \int_{0}^{t} \psi\left(\int_{s}^{+\infty} a(z) f(z, u(z)) d z\right) d s \\
& \leq \frac{1}{1+t} \int_{0}^{1} \psi\left(\int_{s}^{+\infty} a(z) f(z, u(z)) d z\right) d s \\
& +\frac{1}{1+t} \int_{1}^{t} \psi\left(\int_{s}^{+\infty} a(z) f(z, u(z)) d z\right) d s \\
& \leq \frac{1}{1+t} \psi\left(\int_{0}^{+\infty} a(z) f(z, u(z)) d z\right) \\
& +\frac{1}{1+t} \int_{1}^{t} \psi\left(\int_{s}^{+\infty} a(z) f(z, u(z)) d z\right) d s
\end{aligned}
$$

Choose $c>0$ such that $c u_{E} \geq M$ and $\frac{1}{c} \leq N$. Thus $c z u(z) \geq M$ and $\frac{1}{c z} \leq N$ for $z \geq 1$. For $s \geq 1$, we have

$$
\begin{aligned}
\int_{s}^{+\infty} a(z) f(z, u(z)) d z & =\int_{s}^{+\infty} a(z) f\left(z, \frac{1}{c z} c z u(z)\right) d z \\
& \leq \int_{s}^{+\infty}(c z)^{\mu-\lambda}|u(z)|^{\mu} a(z) f(z, 1) d z \\
& \leq \int_{s}^{+\infty}(c z)^{\mu-\lambda}(1+z)^{\mu} u_{E}^{\mu} a(z) f(z, 1) d z \\
& =c^{\mu-\lambda}\left(\int_{s}^{+\infty} z^{\mu-\lambda}(1+z)^{\mu} a(z) f(z, 1) d z\right) u_{E}^{\mu} .
\end{aligned}
$$

Immediately it follows from $(1.4),\left(H_{1}\right)$ and $\left(H_{2}\right)$

$$
\begin{aligned}
\frac{v(t)}{1+t} & \leq \frac{1}{1+t}\left[\psi\left(\int_{0}^{+\infty} a(z) f(z, u(z)) d z\right)\right] \\
& +c^{\frac{\mu-\lambda}{\alpha}} u_{E}^{\frac{\mu}{\alpha}} \frac{1}{1+t}\left[\int_{1}^{t} \psi\left(\int_{s}^{+\infty} z^{\mu-\lambda}(1+z)^{\mu} a(z) f(z, 1) d z\right) d s\right] .
\end{aligned}
$$

Using (3) - (3) and $\left(H_{2}\right)$, we get

$$
t \rightarrow+\infty \lim \frac{v(t)}{1+t}=0 .
$$

We have obviously that $v(0)=0$ and $\left(\phi\left(v^{\prime}\right)\right)^{\prime}(t)=-a(t) f(t, u(t)) \leq 0$. Thus, the facts that $\phi$ is increasing and $\lim _{t \rightarrow \infty} v^{\prime}(t)=0$ imply that $v^{\prime}$ is 
non increasing and nonnegative on $(0,+\infty)$. That is, $v$ is concave. Then $T u \in P$.

Lemma 3.5. Suppose that $\left(H_{1}\right),\left(H_{2}\right)$ and (1.4) hold. Then the operator $T: \bar{P}_{R} \backslash P_{r} \rightarrow P$ is completely continous, for any $0<r<R$ and $P_{r}=$ $\{u \in P, u<r\}$.

\section{Proof.}

- In order to prove that $T$ is continuous. Let $u \in \bar{P}_{R} \backslash P_{r}$ and $\left(u_{n}\right)_{n} \subset$ $\bar{P}_{R} \backslash P_{r}$ be such that $\lim _{n \rightarrow \infty} u_{n}=u$ in $E$. We have $\lim _{n \rightarrow \infty} f\left(t, u_{n}(t)\right)=$ $f(t, u(t)), \forall t \geq 0$.

We have for $s \geq 0$

$$
\begin{aligned}
& \left|\int_{s}^{+\infty} a(z) f\left(z, u_{n}(z)\right) d z-\int_{s}^{+\infty} a(z) f(z, u(z)) d z\right| \\
& \leq \int_{0}^{+\infty} a(z)\left|f\left(z, u_{n}(z)\right)-f(z, u(z))\right| d z \\
& =\int_{0}^{1} a(z)\left|f\left(z, u_{n}(z)\right)-f(z, u(z))\right| d z \\
& +\int_{1}^{+\infty} a(z)\left|f\left(z, u_{n}(z)\right)-f(z, u(z))\right| d z
\end{aligned}
$$

Choose $c>0$ such that $2 c r \leq N, \frac{1}{c} \geq M$, then $c u(z) \leq N$ for $z \in[0,1]$ and $\frac{1}{c} \geq M$.

Similarly to the proof of Lemma 3.4, we get

$$
\begin{aligned}
\int_{0}^{1} a(z)\left|f\left(z, u_{n}(z)\right)-f(z, u(z))\right| d z & \leq c^{\lambda-\mu} r^{\lambda} I_{1}+c^{\lambda-\mu} r^{\lambda} I_{1} \\
& \leq 2 c^{\lambda-\mu} r^{\lambda} I_{1} \\
& \leq+\infty
\end{aligned}
$$

Choose $\delta>0$ such that $\delta R \geq M, \frac{1}{\delta} \leq N$. Thus $\delta z u(z) \geq M$ and $\frac{1}{\delta z} \leq N$, for $z \geq 1$.

$$
\begin{aligned}
& \text { We have } \\
& \qquad \begin{aligned}
\int_{1}^{+\infty}\left|a(z) f\left(z, u_{n}(z)\right)-a(z) f(z, u(z))\right| d z & \leq \delta^{\mu-\lambda} R^{\mu} I_{2}+\delta^{\mu-\lambda} R^{\mu} I_{2} \\
& \leq 2 \delta^{\mu-\lambda} R^{\mu} I_{2} \\
& <+\infty
\end{aligned}
\end{aligned}
$$

The sequence $\left(\int_{s}^{+\infty} a(z) f\left(z, u_{n}(z)\right) d z\right)_{n}$ converge uniformly to $\int_{s}^{+\infty} a(z) f(z, u(z)) d z$ by Lebesgue dominated convergence theorem. The uniform continuity of $\psi$ on compact intervals of $\mathbf{R}^{+}$implies that for all 
$\varepsilon>0$, there exists a positive integer $n_{\varepsilon}$ such that for all $n \geq n_{\varepsilon}$ and for $s \geq 0$, we have

$$
\left|\psi\left(\int_{s}^{+\infty} a(z) f\left(z, u_{n}(z)\right) d z\right)-\psi\left(\int_{s}^{+\infty} a(z) f(z, u(z)) d z\right)\right| \leq \varepsilon .
$$

Then

$$
\begin{aligned}
& T u_{n}-T u_{E} \\
& =t \in \mathbf{R}^{+} \sup \mid \frac{1}{1+t} \int_{0}^{t}\left[\psi\left(\int_{s}^{+\infty} a(z) f\left(z, u_{n}(z)\right) d z\right)\right. \\
& -\psi\left(\int_{s}^{+\infty} a(z) f(z, u(z)) d z\right) d s \\
& \leq t \in \mathbf{R}^{+} \sup \left|\frac{\int_{0}^{t} \varepsilon}{1+t}\right| \\
& =\varepsilon .
\end{aligned}
$$

- We prove that $T\left(\bar{P}_{R} \backslash P_{r}\right)$ is relatively compact.

1. Let $u \in \bar{P}_{R} \backslash P_{r}$ and we show that $T\left(\bar{P}_{R} \backslash P_{r}\right)$ is uniformly bounded. From (3) and (3), we get

$$
\begin{aligned}
T u_{E} & =t \in \mathbf{R}^{+} \sup \left[\frac{1}{1+t} \int_{0}^{t} \psi\left(\int_{s}^{+\infty} a(z) f(z, u(z)) d z\right) d s\right] \\
& \leq t \in \mathbf{R}^{+} \sup \left[\frac{t}{1+t}\right] \psi\left(\int_{0}^{+\infty} a(z) f(z, u(z)) d z\right) \\
& =\psi\left(\int_{0}^{+\infty} a(z) f(z, u(z)) d z\right) \\
& <+\infty .
\end{aligned}
$$

This means that $T\left(\bar{P}_{R} \backslash P_{r}\right)$ is uniformly bounded.

2. $\frac{T\left(\bar{P}_{R} \backslash P_{r}\right)}{1+t}$ is almost equicontinous. Let $A>0$ and $t_{1}, t_{2} \in[0, A]$ with $t_{1}<t_{2}$, we have for $u \in \bar{P}_{R} \backslash P_{r}$

$$
\begin{aligned}
& \left|\frac{T u\left(t_{1}\right)}{1+t_{1}}-\frac{T u\left(t_{2}\right)}{1+t_{2}}\right| \\
& \leq \frac{1}{1+t_{1}} \int_{t_{1}}^{t_{2}} \psi\left(\int_{s}^{+\infty} a(z) f(z, u(z)) d z\right) d s \\
& +\left|\frac{1}{1+t_{2}}-\frac{1}{1+t_{1}}\right| \int_{0}^{t_{2}} \psi\left(\int_{s}^{+\infty} a(z) f(z, u(z)) d z\right) d s \\
& \leq \int_{t_{1}}^{t_{2}} \psi\left(\int_{s}^{+\infty} a(z) f(z, u(z)) d z\right) d s \\
& +\left|\frac{1}{1+t_{2}}-\frac{1}{1+t_{1}}\right| \int_{0}^{T} \psi\left(\int_{s}^{+\infty} a(z) f(z, u(z)) d z\right) d s .
\end{aligned}
$$


The uniform continuity of the function $t \mapsto \int_{0}^{t} \psi\left(\int_{s}^{+\infty} a(z) f(z, u(z)) d z\right) d s$, on the interval $[0, A]$ imply that $T\left(\bar{P}_{R} \backslash P_{r}\right)$ is equicontinuous on $[0, A]$.

3. $T\left(\bar{P}_{R} \backslash P_{r}\right)$ is equiconvergent at $\infty$.

Let $u \in\left(\bar{P}_{R} \backslash P_{r}\right)$, we have

$$
\begin{aligned}
\left|\frac{T u(t)}{1+t}-t \rightarrow+\infty \lim \frac{T u(t)}{1+t}\right| & =\frac{T u(t)}{1+t} \\
& \leq \frac{1}{1+t} \int_{0}^{t} \psi\left(\int_{s}^{+\infty} a(z) f(z, u(z)) d z\right) d s
\end{aligned}
$$

This together with hypothesis $\left(H_{2}\right)$, we have $\lim _{t \rightarrow \infty} \frac{T u(t)}{1+t}=0$.

As a result $\lim _{t \rightarrow \infty}\left|\frac{T u(t)}{1+t}-t \rightarrow+\infty \lim \frac{T u(t)}{1+t}\right|=0$.

Therefore, we deduce from Lemma 2.5 that $T\left(\bar{P}_{R} \backslash P_{r}\right)$ is relatively compact in $E$ and we conclude by Definition 2.2 that $T$ is a completely continuous operator.

Lemma 3.6. Assume that $\left(H_{1}\right)$ and $\left(H_{2}\right)$ hold. Then $u$ is a positive solution to problem (1.2) if and only if $u$ is a positive fixed point of $T$.

Proof. Let $u$ is a positive solution to problem (1.2). By integration, we have:

$$
\int_{s}^{+\infty} \phi\left(u^{\prime}(r)\right) d r=\int_{s}^{+\infty} a(r) f(r, u(r)) d r .
$$

Imply

$$
\phi\left(u^{\prime}\right)(s)=\int_{s}^{+\infty} a(r) f(r, u(r)) d r .
$$

Consequently, $u^{\prime}(s)=\psi\left(\int_{s}^{+\infty} a(r) f(r, u(r)) d r\right)$.

Integrating between 0 and $t$, we obtain

$$
\int_{0}^{t} u^{\prime}(r) d r=\int_{0}^{t} \psi\left(\int_{s}^{+\infty} a(r) f(r, u(r)) d r\right) d s .
$$

Leads to $u(t)=T u(t)$, which imply that $u$ is a fixed point of $T$.

Inversely, let $u$ a fixed point of $T$. We have

$$
u(t)=\int_{0}^{t} \psi\left(\int_{s}^{+\infty} a(r) f(r, u(r)) d r\right) d s
$$

See that $u(0)=0$. By derivating with respect to $t$, we get

$$
u^{\prime}(t)=\psi\left(\int_{t}^{+\infty} a(r) f(r, u(r)) d r\right) .
$$


See that $t \rightarrow+\infty \lim u^{\prime}(t)=0$. Consequently, we have

$$
\phi\left(u^{\prime}\right)(t)=\int_{t}^{+\infty} a(r) f(r, u(r)) d r .
$$

Derivating, we get that $u$ is a positive solution to problem (1.2).

This completes the proof.

Lemma 3.7. Assume that (1.4) and $\left(H_{1}\right)-\left(H_{3}\right)$ hold with $0<\mu<\alpha$. Then any positive solution $u$ of problem (1.2) is unbounded.

Proof. Let $u$ be a positive solution of problem (1.2). We have from Lemma that

$$
\begin{aligned}
u(x) & =\int_{0}^{x} \psi\left(\int_{s}^{+\infty} a(z) f(z, u(z)) d z\right) d s \\
& \geq x \psi\left(\int_{x}^{+\infty} a(z) f(z, u(z)) d z\right)
\end{aligned}
$$

Choose $c>0$ such that $c u(z) \leq N$ for $z \geq 1$ and $\frac{1}{c} \geq M$. Thus, for $x \in[1,+\infty)$, we have

$$
\begin{aligned}
\psi\left(\int_{x}^{+\infty} a(z) f(z, u(z)) d z\right) & =\psi\left(\int_{x}^{+\infty} a(z) f\left(z, c u(z) \frac{1}{c}\right) d z\right) \\
& \geq \psi\left(\int_{x}^{+\infty}|c u(z)|^{\mu} c^{-\lambda} a(z) f(z, 1) d z\right) \\
& \geq \psi\left(\int_{x}^{+\infty} c^{\mu-\lambda} u^{\mu}(x) a(z) f(z, 1) d z\right) \\
& \geq u^{\frac{\mu}{\alpha}}(x) c^{\frac{\mu}{\alpha}-\frac{\lambda}{\beta}} \psi\left(\int_{x}^{+\infty} a(z) f(z, 1) d z\right) .
\end{aligned}
$$

Consequently

$$
u(x)^{1-\frac{\mu}{\alpha}} \geq c^{\frac{\mu}{\alpha}-\frac{\lambda}{\beta}} x \psi\left(\int_{x}^{+\infty} a(z) f(z, 1) d z\right) .
$$

Suppose that $u$ is bounded and let

$$
l_{\infty}=\lim _{x \rightarrow \infty} u(x)>0 .
$$

Using the condition $\left(H_{3}\right)$, we have the contradiction

$$
\infty>l_{\infty}^{1-\frac{\mu}{\alpha}}=\lim _{x \rightarrow \infty} u(x)^{1-\frac{\mu}{\alpha}} \geq c^{\frac{\mu}{\alpha}-\frac{\lambda}{\beta}} \lim _{x \rightarrow \infty} x \psi\left(\int_{x}^{+\infty} a(z) f(z, 1) d z\right)=+\infty .
$$

which imply that $u$ is unbounded and this proves Lemma.

Lemma 3.8. Assume that $(1.4),\left(H_{1}\right),\left(H_{2}\right)$ and the following hypothesis hold 


$$
\lim _{x \rightarrow \infty} x^{\frac{\alpha+\lambda}{\alpha}} \psi\left(\int_{x}^{2 x} \frac{a(z)}{z^{\mu}} f(z, 1) d z\right)=+\infty
$$

where $\lambda \in(-\alpha, 0)$. Then any positive solution $u$ of problem (1.2) is unbounded.

Proof. Let $u$ be a positive solution of the problem (1.2).

Choose $c>0$ such that $c u \geq M$ and $\frac{1}{c} \leq N$. It follows for $z \geq 1$ that $c z u(z) \geq M$ and $\frac{1}{c z} \leq N$.

for all $x \in[1,+\infty)$, we have from lemma 3 ,that

$$
\begin{aligned}
u(2 x) & =\int_{0}^{2 x} \psi\left(\int_{s}^{+\infty} a(z) f(z, u(z)) d z\right) d s \\
& \geq \int_{0}^{x} \psi\left(\int_{s}^{+\infty} a(z) f(z, u(z)) d z\right) d s \\
& \geq x \psi\left(\int_{x}^{+\infty} a(z) f(z, u(z)) d z\right) \\
& \geq x \psi\left(\int_{x}^{2 x} a(z) f(z, u(z)) d z\right) .
\end{aligned}
$$

It follows that

$$
\begin{aligned}
u(2 x) & \geq x \psi\left(\int_{x}^{2 x} a(z) f(z, u(z)) d z\right) \\
& =x \psi\left(\int_{x}^{2 x} a(z) f\left(z, c z u(z) \cdot \frac{1}{c z}\right) d z\right) \\
& \geq x \psi\left(\int_{x}^{2 x}|c z u(z)|^{\lambda}\left(\frac{1}{c z}\right)^{\mu} a(z) f(z, 1) d z\right) \\
& \geq x \psi\left(\int_{x}^{2 x} c^{\lambda-\mu}(2 x)^{\lambda} u^{\lambda}(2 x) z^{-\mu} a(z) f(z, 1) d z\right) \\
& \geq \frac{1}{2}(2 x)^{\frac{\lambda}{\alpha}+1} c^{\frac{\lambda}{\alpha}-\frac{\mu}{\beta}} u^{\frac{\lambda}{\alpha}}(2 x) \psi\left(\int_{x}^{2 x} a(z) z^{-\mu} f(z, 1) d z\right) .
\end{aligned}
$$

Leads to

$$
u^{1-\frac{\lambda}{\alpha}}(2 x) \geq \Lambda_{\alpha, \beta, \lambda, \mu}(2 x)^{\frac{\lambda}{\alpha}+1} \psi\left(\int_{x}^{2 x} a(z) z^{-\mu} f(z, 1) d z\right)
$$

where $\Lambda_{\alpha, \lambda, \mu}=\frac{c^{\frac{\lambda}{\alpha}-\frac{\mu}{\beta}}}{2}$.

Then from 3.1, we have

$u^{\frac{\alpha-\lambda}{\alpha}}(2 x) \geq \Lambda_{\alpha, \beta, \lambda, \mu}(2 x)^{\frac{\alpha+\lambda}{\alpha}} \psi\left(\int_{x}^{2 x} a(z) z^{-\mu} f(z, 1) d z\right) \longrightarrow+\infty$, as $x \longrightarrow+\infty$.

As in the proof of the previous Lemma, we conclude that $u$ is unbounded. This proves Lemma.

Let for $R>0, P_{R}=P \cap B_{R}\left(0_{E}\right)$ where $B_{R}\left(0_{E}\right)$ is the open ball of radius $R$ centred at $0_{E}$, and let $\partial P_{R}=P \cap \partial B_{R}\left(0_{E}\right)$ be its boundary. 
Theorem 3.9. Assume that (1.4) and $\left(H_{1}\right)-\left(H_{3}\right)$ with $\mu<\alpha$ hold. Then $B V P(1.2)$ has at least one unbounded positive solution.

Proof. In order to make use of Theorem 2.3, let us prove there exists a sufficiently small $r>0$ such that

$$
T u_{E} \geq u_{E}, \forall u \in \partial P_{r}
$$

Let $c=\frac{1}{M}$ and $r=\min \left(\frac{N}{2 c},\left[\frac{1}{4} M^{\frac{\mu}{\beta}-\frac{\lambda}{\alpha}} \psi\left(\int_{\frac{1}{2}}^{1} z^{\mu} a(z) f(z, 1) d z\right)\right]^{\frac{\alpha}{\alpha-\mu}}\right)$.

It follows $c u(z) \leq 2 c r \leq N$ for $u \in \partial P_{r}$ and $z \in[0,1]$.

We have

$$
\begin{aligned}
T u_{E} & =x \in \mathbf{R}^{+} \sup \left|\frac{T u(x)}{1+x}\right| \\
& \geq \frac{T u(1)}{2} \\
& =\frac{1}{2} \int_{0}^{1} \psi\left(\int_{s}^{+\infty} a(z) f(z, u(z)) d z\right) d s \\
& \geq \frac{1}{2} \int_{0}^{\frac{1}{2}} \psi\left(\int_{s}^{1} a(z) f(z, u(z)) d z\right) d s \\
& \geq \frac{1}{2} \int_{0}^{\frac{1}{2}} \psi\left(\int_{s}^{1} c^{\mu-\lambda} u^{\mu}(z) a(z) f(z, 1) d z\right) d s \\
& \geq \frac{1}{4} \psi\left(\int_{\frac{1}{2}}^{1} c^{\mu-\lambda} z^{\mu} u_{E}^{\mu} a(z) f(z, 1) d z\right) \\
& \geq \frac{1}{4} c^{\frac{\lambda}{\alpha}-\frac{\mu}{\beta}} u_{E}^{\frac{\mu}{\alpha}} \psi\left(\int_{\frac{1}{2}}^{1} z^{\mu} a(z) f(z, 1) d z\right) \\
& =\left[\frac{1}{4} M^{\frac{\mu}{\beta}-\frac{\lambda}{\alpha}} \psi\left(\int_{\frac{1}{2}}^{1} z^{\mu} a(z) f(z, 1) d z\right)\right] u_{E}^{\frac{\mu}{\alpha}} \\
& \geq r=u_{E}, \quad \forall u \in \partial P_{r} .
\end{aligned}
$$

Which implies that (3.2) holds.

We show there exists a sufficiently large $R>r$ such that

$$
T u_{E} \leq u_{E}, \forall u \in \partial P_{R}
$$

Choose $R_{1}>r$ satisfying $\frac{2 R_{1}}{N} \geq M$. Let $c>0$ such that $c=\frac{N}{2 R_{1}}$ then $\frac{1}{c} \geq M$.

Choose $R_{2}>R_{1}$ satisfying $R_{2} \geq M N$ and let $R=\max \left(R_{2},\left[\left(\frac{N}{2}\right)^{\frac{\lambda-\mu}{\alpha}} \psi\left(I_{1}+I_{2}\right)\right]^{\frac{\mu}{\mu-\alpha}}\right)>0$. From $\left(H_{1}\right)$ and similarly to prove of Lemma 3.4, we have from (3) and (3) that 


$$
\begin{aligned}
T u_{E} & =x \in \mathbf{R}^{+} \sup \left|\frac{1}{1+x} \int_{0}^{x} \psi\left(\int_{s}^{+\infty} a(z) f(z, u(z)) d z\right) d s\right| \\
& \leq x \in \mathbf{R}^{+} \sup \left|\frac{x}{1+x}\right| \psi\left(\int_{0}^{+\infty} a(z) f(z, u(z)) d z\right) \\
& =\psi\left(\int_{0}^{1} a(z) f(z, u(z)) d z+\int_{1}^{+\infty} a(z) f(z, u(z)) d z\right) \\
& \leq \psi\left(\left(\frac{N}{2}\right)^{\lambda-\mu} R_{1}^{\mu} I_{1}+2^{\lambda-\mu}\left(\frac{N}{2}\right)^{\lambda-\mu} R_{2}^{\mu} I_{2}\right) .
\end{aligned}
$$

This implies

$$
\begin{aligned}
T u_{E} & \leq \psi\left(\left(\frac{N}{2}\right)^{\lambda-\mu} R^{\mu}\left(I_{1}+I_{2}\right)\right) \\
& \leq\left(\frac{N}{2}\right)^{\frac{\lambda-\mu}{\alpha}} \psi\left(I_{1}+I_{2}\right) R^{\frac{\mu}{\alpha}} \\
& \leq R^{1-\frac{\mu}{\alpha}} R^{\frac{\mu}{\alpha}} \\
& =R=u_{E} .
\end{aligned}
$$

This implies that (3.3) hold.

We deduce from ii) of Theorem 2.3 that $T$ admits a fixed point $u \in K$ with $r<\|u\|_{E}<R$ which is, by Lemmas 3.6 and 3.7 a positive unbounded solution to Problem (1.2).

Corollary 3.10. Assume that (1.4), $\left(H_{1}\right),\left(H_{2}\right),(3.1)$ hold and $\mu<\alpha$. Then BVP (1.2) has at least one unbounded positive solution.

The next theorem generates a multiplicity result for BVP (1.2).

Theorem 3.11. Assume that $(1.4)$ and $\left(H_{1}\right)-\left(H_{3}\right)$ hold. In addition suppose that

$$
\begin{aligned}
& \left(H_{4}\right) \quad c^{\lambda-\mu} I_{1}+\delta^{\mu-\lambda} I_{2}<\phi(1), \\
& \text { where } \quad c=\min \left(\frac{N}{2}, \frac{1}{M}\right), \delta=\max \left(\frac{1}{N}, M\right) .
\end{aligned}
$$

$\left(H_{5}\right) \quad$ There exists $\mu_{*} \in \mathbf{R}$ such that $0<\mu_{*}<\beta$ satisfying

$$
f(t, c u) \geq c^{\mu_{*}} \phi(u), \quad c \geq M .
$$

Then BVP (1.2) has at least two unbounded positive solutions $u, v \in P$ such that

$$
0<u<1<v<\infty .
$$


Proof. Similarly to the proof of the previous theorem, we can prove that there exists a sufficiently small $r \in(0,1)$ to guarantee $T u_{E} \geq u_{E}, \forall u \in \partial P_{r}$. Secondly, we show that

$$
T u_{E}<u_{E}, \forall u \in \partial P_{1} .
$$

Choose $c>0$ such that $2 c \leq N, \frac{1}{c}>M$ and choose $\delta>0$ such that $\delta \geq M, \frac{1}{\delta} \leq N$. Thus $\delta z u(z) \geq M$ and $\frac{1}{\delta z} \leq N$.

We have

$$
\begin{aligned}
T u & =x \in \mathbf{R}^{+} \sup \left|\frac{1}{1+x} \int_{0}^{x} \psi\left(\int_{s}^{+\infty} f(z, u(z)) d z\right) d s\right| \\
& \leq \psi\left(\int_{0}^{+\infty} f(z, u(z)) d z\right) \\
& =\psi\left(\int_{0}^{1} a(z) f(z, u(z)) d z+\int_{1}^{+\infty} f(z, u(z)) d z\right) \\
& =\psi\left(\int_{0}^{1} a(z) f\left(z, \frac{1}{c} c u(z)\right) d z+\int_{1}^{+\infty} a(z) f\left(z, \frac{1}{\delta z} \delta u(z)\right) d z\right) \\
& \leq \psi\left(c^{\lambda-\mu} u^{\lambda} \int_{0}^{1} z^{\lambda} a(z) f(z, 1) d z+\delta^{\mu-\lambda} u^{\mu} \int_{1}^{+\infty} z^{\mu-\lambda}(1+z)^{\mu} a(z) f(z, 1) d z\right) \\
& =\psi\left(c^{\lambda-\mu} \int_{0}^{1} z^{\lambda} a(z) f(z, 1) d z+\delta^{\mu-\lambda} \int_{1}^{+\infty} z^{\mu-\lambda}(1+z)^{\mu} a(z) f(z, 1) d z\right) \\
& <\psi(\phi(1)) \\
& =1=u_{E} .
\end{aligned}
$$

This implies that (3.4) holds. We deduce from ii) of Theorem 2.3 that $T$ admits a fixed point $u \in K$ with $r<\|u\|_{E}<1$ which is, by Lemmas 3 and 3.7 a positive unbounded solution to Problem (1.2) .

Now, we show there exists a sufficiently large $R>1$ such that $T u_{E} \geq$ $u_{E}, \forall u \in \partial P_{R}$, where

$R=\max \left(M N,\left(\frac{5}{6} \psi^{-}(\phi(1)) \psi^{-}\left(N^{\frac{\mu-\mu_{*}}{\beta}}\right) \psi\left(\int_{5}^{10} z^{-\lambda_{*}} a(z) d z\right)\right)^{\frac{\beta}{\beta-\mu_{\star}}}\right)$.

Let $c=\frac{1}{N}$ and $u \in \partial P_{R}$. We have $z u(z) \geq u_{E}=R$ for $z \in[1,+\infty)$. Thus $c z u(z) \geq M$ and $\frac{1}{z c}=\frac{N}{z} \leq N$.

Using $\left(H_{5}\right)$ and (1.4), we have 


$$
\begin{aligned}
T u_{E} & =x \in \mathbf{R}^{+} \sup \left|\frac{T u(x)}{1+x}\right| \geq \frac{T u(5)}{6} \\
& \geq \frac{1}{6} \quad \int_{0}^{5} \psi\left(\int_{s}^{+\infty} a(z) f(z, u(z)) d z\right) d s \\
& =\frac{5}{6} \psi\left(\int_{5}^{10} a(z) f(z, u(z)) d z\right) \\
& =\frac{5}{6} \psi\left(\int_{5}^{10} a(z) f\left(z, \frac{1}{c z} c z u(z)\right) d z\right) \\
& \geq \frac{5}{6} \psi\left(\int_{5}^{10}|c z u(z)|^{\mu_{*}}\left(\frac{1}{c z}\right)^{\mu} a(z) \phi(1) d z\right) \\
& =\frac{5}{6} \psi\left(\int_{5}^{10} c^{\mu_{*}-\mu} z^{-\mu} u_{E}^{\mu_{*}} a(z) \phi(1) d z\right) \\
& \geq \frac{5}{6} \psi^{-}\left(c^{\frac{\mu_{*}-\mu}{\beta}}\right) u_{E}^{\frac{\mu_{*}}{\beta}} \psi\left(\int_{5}^{10} z^{-\mu} a(z) \phi(1) d z\right) \\
& \geq \frac{5}{6} \psi^{-}(\phi(1)) \psi^{-}\left(N^{\frac{\mu-\mu_{*}}{\beta}}\right) R^{\frac{\mu_{*}}{\beta}} \psi\left(\int_{5}^{10} z^{-\mu} a(z) d z\right) \\
& \geq R .
\end{aligned}
$$

We deduce from i) of Theorem 2.3 that $T$ admits a fixed point $v \in K$ with $1<\|v\|_{E}<R$ which is, by Lemmas 3.6 and 3.7 a positive unbounded solution to Problem (1.2). This completes the proof.

Remark 3.12. In the previous theorem, we can get the same results if we replace the condition (3.1) by the hypothesis $\left(H_{3}\right)$.

Example 3.13. Consider the boundary value problem (1.2) with $\phi(u)=$ $u^{p-1}+u^{q-1}, 2<p<q, a(t)=\frac{1}{1+t}$ and $f(t, u)=\frac{1}{\sqrt[8]{u}(1+t)}$. Clearly (1.4) is satisfied with $\alpha=p-1, \beta=q-1$ and $\left(H_{1}\right)$ is satisfied with $\mu=\frac{1}{8}$ and $\lambda=-\frac{1}{8}$.

Straightforward computations lead to

$$
\int_{0}^{1} t^{\lambda} a(z) f(t, 1) d t=\int_{0}^{1} t^{-\frac{1}{8}} \frac{1}{(1+t)^{2}} d t \leq \int_{0}^{1} \frac{d t}{\sqrt[8]{t}}<\infty .
$$

and for $s>1$, we have:

$$
\int_{s}^{+\infty} t^{\mu-\lambda}(1+t)^{\mu} a(t) f(t, 1) d t \leq \int_{s}^{+\infty} \frac{d t}{(1+t)^{\frac{13}{8}}}<\infty .
$$

Leads

$$
\int_{s}^{+\infty} \frac{d t}{(1+t)^{\frac{13}{8}}}=\frac{8}{5} \frac{1}{(1+s)^{\frac{5}{8}}}
$$


Thus, we get

$$
\int_{1}^{t} \psi\left(\int_{s}^{+\infty} z^{\mu-\lambda}(1+z)^{\mu} a(z) f(z, 1) d z\right) d s
$$

Then we have $\leq \psi\left(\frac{8}{5}\right) \int_{1}^{t}\left(\frac{1}{1+s}\right)^{\frac{5}{8(q-1)}} d s$

$$
=\left(1-\frac{5}{8(q-1)}\right) \psi\left(\frac{8}{5}\right)\left[(1+t)^{1-\frac{5}{8(q-1)}}-2^{1-\frac{5}{8(q-1)}}\right] \text {. }
$$

$$
\lim _{t \rightarrow \infty} \frac{1}{1+t}\left[(1+t)^{1-\frac{5}{8(q-1)}}-2^{1-\frac{5}{8(q-1)}}\right]=0,
$$

and thus $\left(H_{2}\right)$ is satisfied.

$$
\begin{aligned}
& \text { By simple computations we get } \\
& \qquad \begin{aligned}
\lim _{x \rightarrow \infty} x \psi\left(\int_{x}^{+\infty} a(z) f(z, 1) d z\right) & =\lim _{x \rightarrow \infty} x \psi\left(\frac{1}{1+x}\right) \\
& =\lim _{x \rightarrow \infty} x\left(\frac{1}{1+x}\right)^{\frac{1}{p-1}} \\
& \geq \lim _{x \rightarrow \infty} 2^{-\frac{1}{p-1}} x^{\frac{p-2}{p-1}}=+\infty .
\end{aligned}
\end{aligned}
$$

Thus $\left(H_{3}\right)$ is satisfied.

Thus, we conclude from Theorem 3.9 that Problem (1.2) has at least one positive unbounded solution.

Example 3.14. Consider the boundary value problem (1.2) with $\phi(u)=$ $u^{3}+u, a(t)=\frac{1}{2^{8}} \frac{1}{(1+t)^{9}}$ and $f(t, u)=\frac{1}{\sqrt{u}(1+t)^{2}}+u^{3}+u$. Clearly (1.4) is satisfied with $\alpha=1, \beta=3$ and $\left(H_{1}\right)$ is satisfied with $\mu=3$ and $\lambda=-\frac{1}{2}$.

Straightforward computations lead to

$$
\int_{0}^{1} t^{-\frac{1}{2}} a(t) f(t, 1) d t \leq \frac{1}{2^{6}} \int_{0}^{1} \frac{d t}{\sqrt{t}} \leq \frac{1}{2^{5}}<\infty .
$$

and for all $s \geq 1$, we have:

$$
\int_{s}^{+\infty} t^{\mu-\lambda}(1+t)^{\mu} a(t) f(t, 1) d t \leq \frac{1}{2^{6}} \int_{s}^{+\infty} \frac{d t}{(1+t)^{2}}=\frac{1}{2^{6}} \frac{1}{(1+s)} .
$$

Leads

$$
\int_{1}^{+\infty} t^{\mu-\lambda}(1+t)^{\mu} a(t) f(t, 1) d t \leq \frac{1}{2^{7}}<\infty .
$$

Then we have

$$
\begin{aligned}
\int_{1}^{t} \psi\left(\int_{s}^{+\infty} t^{\mu-\lambda}(1+t)^{\mu} a(t) f(t, 1) d t\right) d s & \leq \int_{1}^{t} \psi\left(\frac{1}{2^{6}} \frac{1}{(1+s)}\right) d s \\
& \leq \int_{1}^{t} \psi\left(\frac{1}{2^{6}}\right)\left(\frac{1}{(1+s)}\right)^{\frac{1}{3}} d s \\
& =\frac{3}{2}\left[(1+t)^{\frac{2}{3}}-2^{\frac{2}{3}}\right] .
\end{aligned}
$$


Thus, we get

$$
\begin{gathered}
\lim _{t \rightarrow \infty} \frac{1}{1+t} \int_{1}^{t} \psi\left(\int_{s}^{+\infty} t^{\mu-\lambda}(1+t)^{\mu} a(t) f(t, 1) d t\right) d s \\
\leq \lim _{t \rightarrow \infty} \frac{1}{1+t} \int_{1}^{t} \psi\left(\frac{1}{2^{6}}\left(\frac{1}{1+s}\right)\right) d s=0,
\end{gathered}
$$

and thus $\left(\mathrm{H}_{2}\right)$ is satisfied.

By simple computations we get $c=\frac{1}{2}, \delta=1$ and

$$
2^{\frac{7}{2}} I_{1}+I_{2} \leq 2^{4} \frac{1}{2^{5}}+\frac{1}{2^{7}}=\frac{1}{2}+\frac{1}{2^{7}}<1<2=\phi(1) .
$$

Furthermore, for $c>1$ we have $\mu^{\star}=1$, because

$$
f(t, c u) \geq c \phi(u) .
$$

Thus $\left(H_{4}\right)$ and $\left(H_{5}\right)$ are satisfied.

Thus, we conclude from Theorem 3.11 that Problem (1.2) has at least two positive unbounded solution.

Acknowledgement. The authors would like to thank the Ministry of Higher Education and Scientific Research and there laboratories, Dynamic of Engines and Vibroacoustic Laboratory, FSI, UMBB, Boumerdes and Fixed Point Theory and Applications, ENS, Kouba for supporting this work.

\section{References}

[1] R. P. Agarwal and D. O’Regan, "Second-Order Boundary Value Problems of Singular Type", Journal of mathematical analysis and applications, vol. 226, no. 2, pp. 414-430, 1998. doi: 10.1006/jmaa.1998.6088

[2] R. P. Agarwal and D. O’Regan, Infinite interval problems for differential, difference and integral equations. Dordrecht: Kluwer Academic Publishers, 2001.

[3] C. Corduneanu, Integral equations and stability of feedback systems. New York: Academic Press, 1973. 
[4] S. Djebali and O. Saifi, "Positive solutions for singular $\phi$-Laplacian BVPs on the positive half-line", Electronic journal of qualitative theory of differential equations, no. 56, pp. 1-24, 2009. doi: 10.14232/ ejqtde. 2009.1.56

[5] D. Guo and V. Lakshmikantham, Nonlinear problems in abstract cones. San Diego: Academic Press, 1988.

[6] P. Kang and Z. Wei, "Multiple solutions of second-order three-point singular boundary value problems on the half-line", Applied mathematics and computation, vol. 203, no. 2, pp. 523-535, 2008. doi: 10.1016/j.amc.2008.04.057

[7] S. Liang and J. Zhang, "The existence of countably many positive solutions for nonlinear singular m-point boundary value problems on the half-line", Journal of computational and applied mathematics, vol. 222, no. 2, pp. 229-243, 2008. doi: 10.1016/j.cam.2007.10.062

[8] S. Liang and J. Zhang, "The existence of countably many positive solutions for one-dimensional p-Laplacian with infinitely many singularities on the half-line", Applied mathematics and computation, vol. 201, no. 1-2, pp. 210-220, 2008. doi: 10.1016/ j.amc.2007.12.016

[9] D. O'Regan, B. Yan, and R. P. Agarwal, "Solutions in weighted spaces of singular boundary value problems on the half-line", Journal of computational and applied mathematics, vol. 205, no. 2, pp. 751-763, 2007. doi: 10.1016/j.cam.2006.02.055

[10] B. Yan, D. O’Regan, and R. P. Agarwal, “Unbounded solutions for singular boundary value problems on the semi-infinite interval: Upper and lower solutions and multiplicity", Journal of computational and applied mathematics, vol. 197, no. 2, pp. 365-386, 2006. doi: 10.1016/j.cam. 2005.11.010

[11] Y. Liu, "Existence and unboundedness of positive solutions for singular boundary value problems on half-line", Applied mathematics and computation, vol. 144, no. 2-3, pp. 543-556, 2003. doi: 10.1016/ s00963003(02)00431-9 


\section{Chahira Attia}

Dynamic of Engines and Vibroacoustic Laboratory,

Faculty of Sciences

University of Boumerdes,

Boumerdes,

Algeria

e-mail: c.attia@univ-boumerdes.dz

\section{Salima Mechrouk}

Faculty of Sciences,

University of Boumerdes,

Boumerdes,

Algeria

e-mail:s.mechrouk@univ-boumerdes.dz

Corresponding author

and

\section{Ouiza Saifi}

Departement of Economics,

Faculty of Economic and Management Sciences,

Algiers University 3,

Algeria

e-mail: saifi-rayen@hotmail.dz 